Original Contribution

\title{
VIDEO-ASSISTED THORACOSCOPIC DIAGNOSIS OF TRAPPED LUNG IN MALIGNANT PLEURAL EFFUSIONS
}

\author{
P. Bonev*, Iv. Novakov, I. Dimitrov, M. Vazheva, A. Uchikov \\ Department of General and Thoracic Surgery, Medical University- Plovdiv, \\ University Hospital “St. George"-Plovdiv, Bulgaria
}

\begin{abstract}
PURPOSE. The aim of this study is to present the role of video-assisted thoracoscopy to assess the ability of the lung to expand in malignant pleural effusions. METHODS. The survey was conducted for a 3-year period in 13 patients with histologically diagnosed malignant pleural effusion and trapped lung. In each of the patients video-assisted thoracoscopy was performed. RESULTS. Two reasons for trapped lung were identified. In 10 of the patients it was due to multiple metastatic nodules. In 3 cases the cause was a diffuse fibrotic involvement of the lung resulting from the metastatic process.CONCLUSIONS. Our research confirmed two causes of trapped lung in malignant pleural effusions: multiple metastases on the lung surface and diffuse lung fibrosis, induced by the metastatic process, with the increased role of multiple metastases on the lung surface.
\end{abstract}

Key words: malignant pleural effusion; thoracoscopy, trapped lung; chest tube; pleurodesis

\section{INTRODUCTION}

'Trapped lung' is a condition of incomplete expansion of the lungs and presence of a residual cavity. This condition results from pathologic processes involving the visceral pleura in malignant and non-malignant diseases. Trapped lung is frequently found in patients with malignant pleural effusions as a result of metastases to the visceral pleura. Trapped lung due to malignant pleural effusion (MPE) is a serious therapeutic problem in the clinical practice of the thoracic surgeons (1-6). The most frequent causes for trapped lung in non-malignant diseases are empyema, parapneumonic effusions, chronic heart failure, uremia. The conditions needed for the formation of trapped lung are long-term persistence of a pleural effusion resulting in the creation of a fibrous visceral pleural peel that restricts the lung to re-expand $(2,3,5)$. The aim of the study is to present the role of videoassisted thoracoscopy in the assessment of the ability of the lungs to expand in malignant pleural effusions.

\section{MATERIAL AND METHODS}

The survey was conducted for a period of 3 years, from January 2012 to December 2014 in the Department of Thoracic Surgery, Medical University of Plovdiv. The results of 13 patients with histologically diagnosed malignant pleural effusion and trapped lung are presented. The distribution according to primary tumor location is presented in Table $\mathbf{1}$.

Table 1. Distribution of patients according to primary tumor location.

\begin{tabular}{|c|c|}
\hline Primary tumor location & Patients \\
\hline 1. Lung cancer & 4 \\
\hline 2. Breast cancer & 2 \\
\hline 3. Cancer of unknown primary & 2 \\
\hline $\begin{array}{l}\text { 4. Urogenital cancers ( renal, ovarian, uterine } \\
\text { cancer) }\end{array}$ & 3 \\
\hline 5. Gastrointestinal cancers & 1 \\
\hline 6. Myeloma & 1 \\
\hline
\end{tabular}

*Correspondence to: Petar Bonev, Medical

University-Plovdiv; Department of General and

Thoracic Surgery; e-mail: petar_bonev@abv.bg 
The imaging techniques used to diagnose malignant pleural effusions are:

1. Chest radiograph - performed in all patients preoperatively and postoperatively.

2. Transthoracic ultrasonography - performed in all patients.

3. Computed tomography (CT) of the chestperformed in 11 patients.

\section{Diagnostic interventional methods.}

We performed in all of 13 patients included in the study:

1. Thoracocentesis with pleural fluid cytology.

2. Fibrobronchoscopy.

3. Video-assisted thoracoscopic surgery (VATS) and pleural biopsy.

\section{Methods for treatment of patients with MPE and trapped lung.}

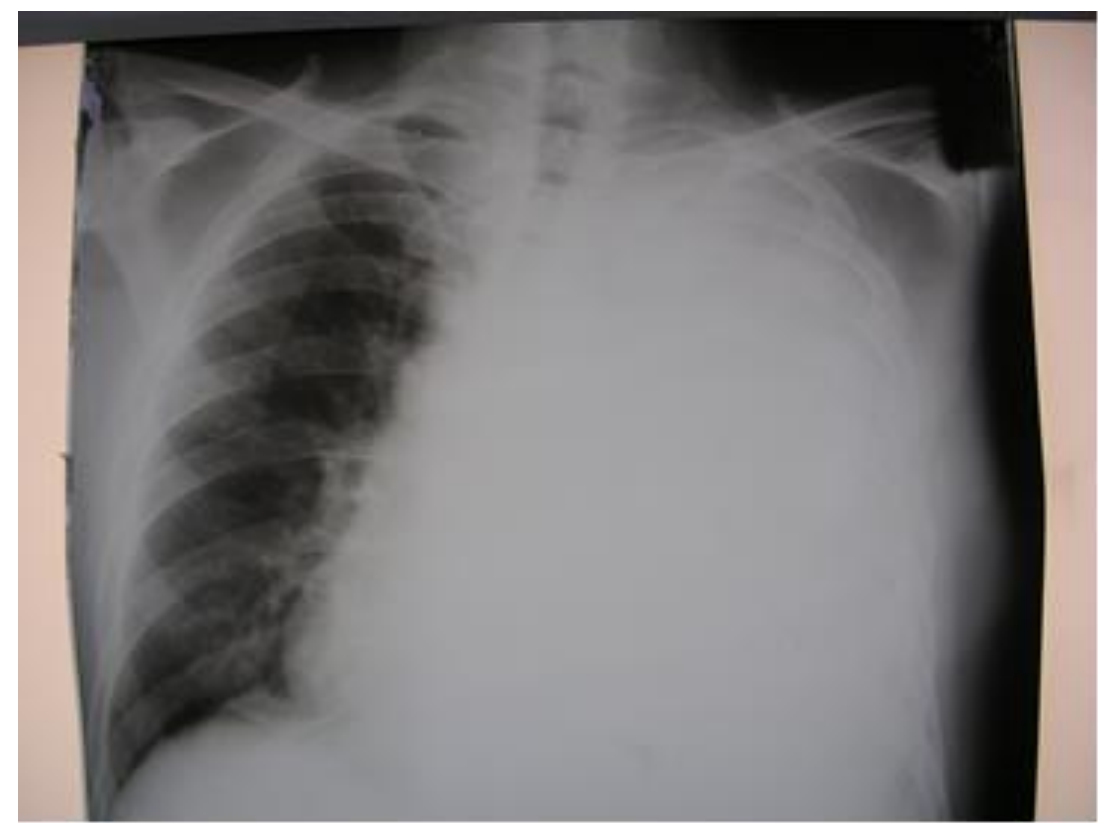

Figure 1. Radiograph of a patient with left-sided MPE and trapped lung (massive pleural effusion reaching the degree of total involvement of the corresponding hemithorax.)

The radiograph performed after the evacuation of the pleural effusion shows unexpanded lung with pneumothorax ex vacuo, the chest tube is visible in the thorax (Figure 2). The performed chest $\mathrm{CT}$ in 11 of the patients showed pleural effusion on the same side as the metastatic disease. In 4 of the patients there were enlarged mediastinal lymph nodes. In 6 of the patients there was parietal pleura thickening (more than $10 \mathrm{~mm}$ ) with metastatic nodules (Figure 3). The presence of a pleural effusion makes the interpretation of the underlying lung pathology much harder.
The patients underwent evacuation of the pleural effusion and subsequent insertion of a permanent chest tube. We used chest tubes ranging from 24 - French to 30 - French in size. The insertion of the tube was conducted under video-assisted thoracoscopic control.

\section{RESULTS}

Clinical presentation: the patients included in the survey have symptomatic malignant pleural effusions with clinical presentation of varying degrees of dyspnea as well as varying degrees of restriction of the physical activities.

Results of the imaging and interventional tests: the chest radiograph performed before the operation showed pleural effusion on the same side as the metastatic disease. In most of the cases the pleural effusion is massive reaching the degree of total involvement of the corresponding hemithorax (Figure 1). 


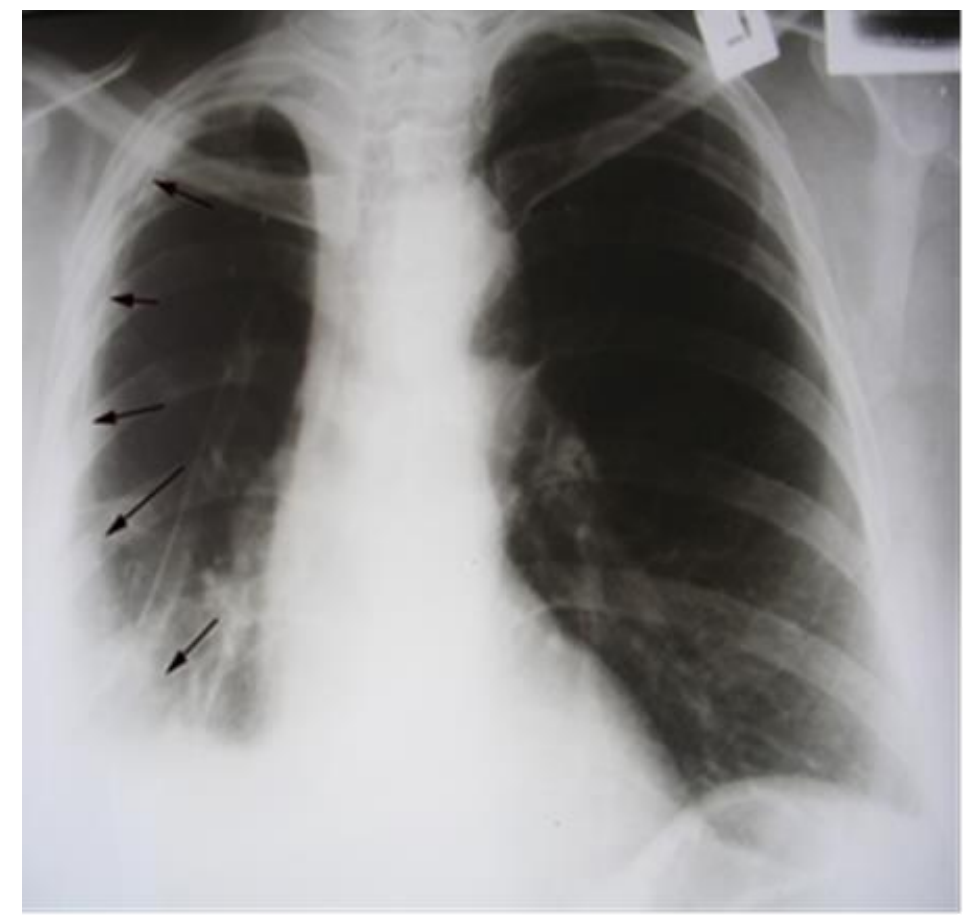

Figure 2. Radiograph of a patient with trapped right lung after evacuation of the effusion (drainage after pneumothorax ex vacuo; the arrows mark the surface of the lung.)

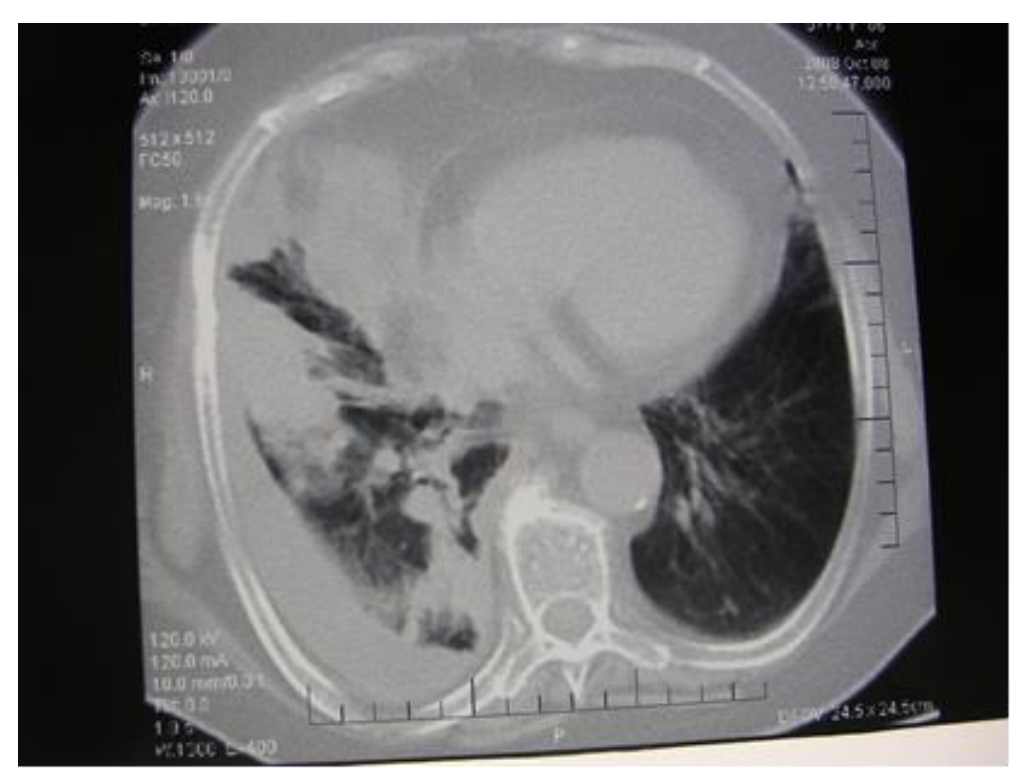

Figure 3. Chest CT of a patient with right MPE and trapped lung (pleural effusion and metastatic nodules on the visceral pleura).

The changes in the pleural cavity were diagnosed through VATS i.e. pleural effusions, metastatic nodules on the parietal and visceral pleura or the diaphragm, fibrotic thickening of the visceral pleura. We took pleural biopsies in all of the patients with following histological analysis. The primary location of the cancer and the cause for MPE were proved based on the cytological and histological evaluation presented on tab. 1. In $9(69 \%)$ of the cases the cause for trapped lung was multiple metastatic nodules on the visceral pleura (Figure 4). The $4(31 \%)$ of the cases the cause for incomplete expansion was fibrotic thickening of the visceral pleura (Figure 5). In these cases the histological analysis of the fibrotic tissue showed metastatic involvement.

Results after drainage of MPE and follow-up: the follow-up period is 30 days after the evacuation of the pleural effusion, with no mortality for this period. Full control of the dyspnea is established in $3(23 \%)$ of the patients. The rest 10 patients $(77 \%)$ report incomplete or partial control regarding the dyspnea with registered improvement of the physical activity. In one patient (7\%) we registered as a complication cellulitis around the chest tube and in 1 patient (7\%)- infection of the pleural cavity and empyema. 


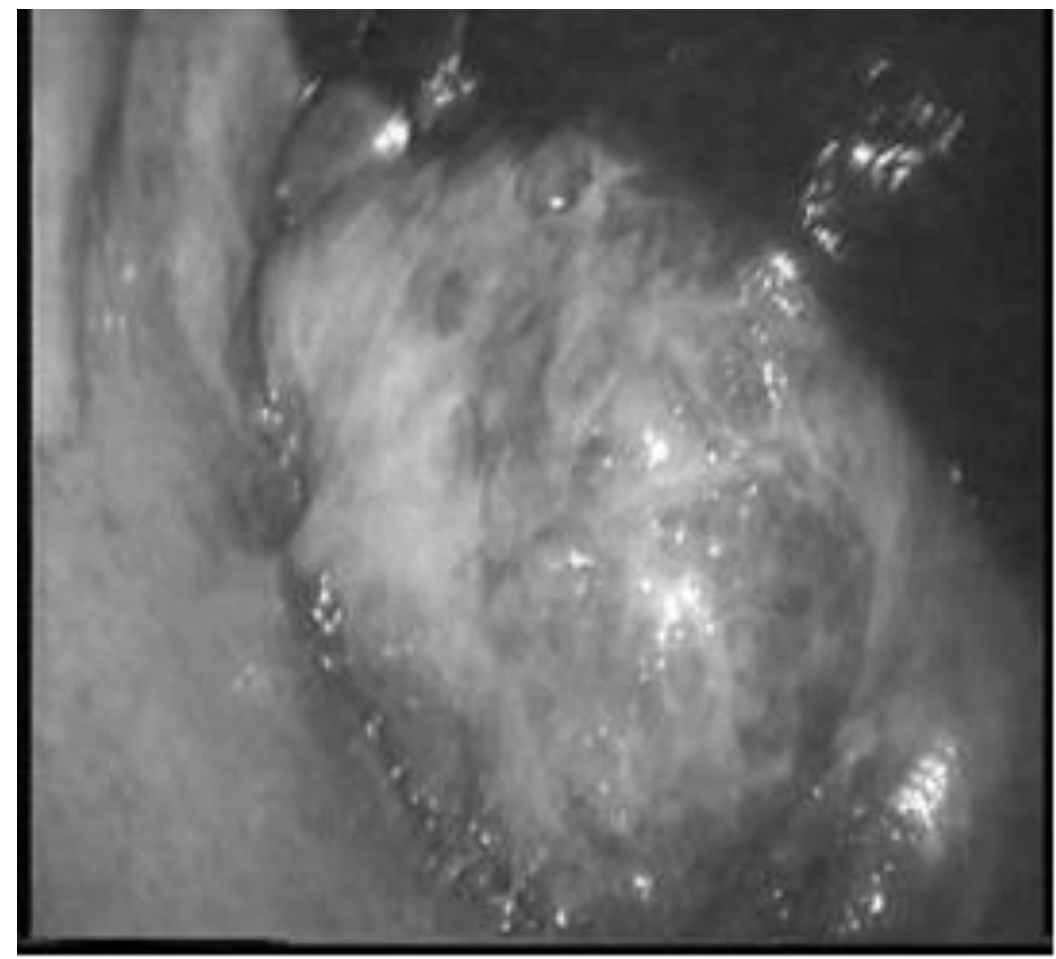

Figure 4. Video-assisted thoracoscopic view of trapped lung by metastatic nodules on the visceral pleura.

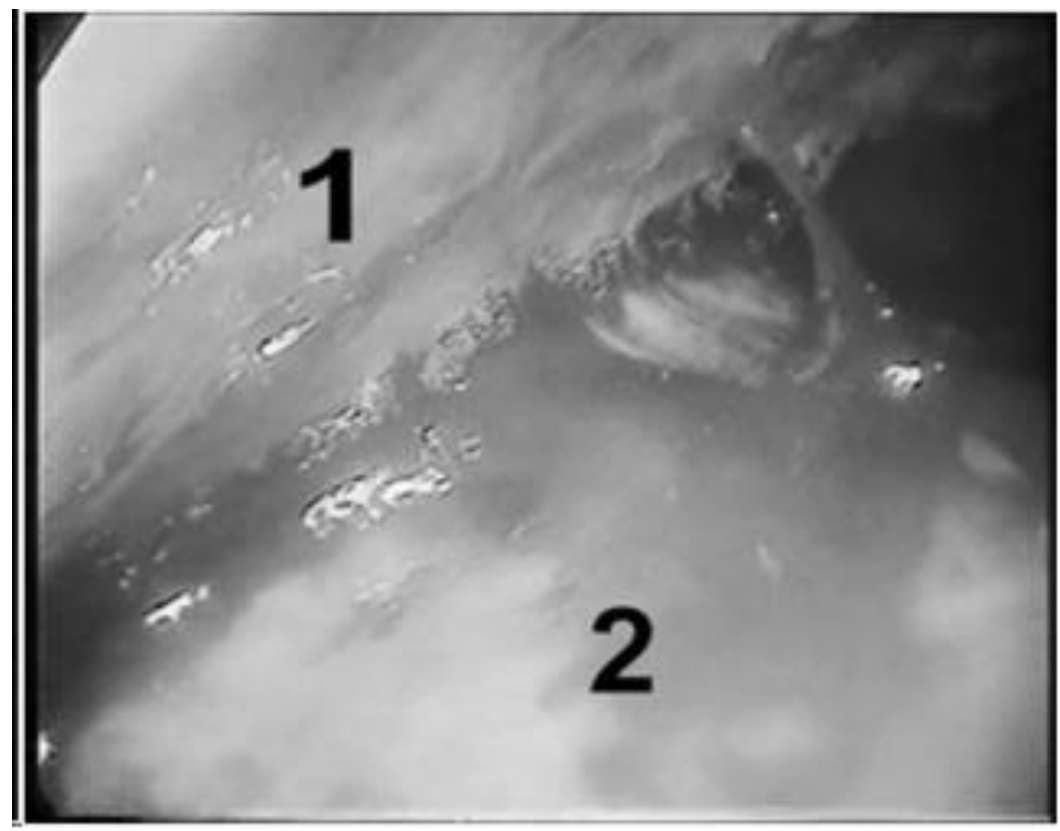

Figure 5. Video-assisted thoracoscopic view of trapped lung by visceral fibrotic membrane (2).

\section{DISCUSSION}

The term "trapped lung" in patients with MPE is a condition of incomplete expansion of the lung due to restriction by the visceral pleura as a result of pleural metastases. Another frequent cause for incomplete expansion of the lung is endobronchial tumour obstruction leading to obstructive atelectasis. The latter is the reason for the formation of pleural effusions with the characteristics of transudate with no cancer cells in it. These pleural effusions are called 'paramalignant' $(3,7)$.
The endobronchial obstruction in patients with MPE and trapped lung is ruled out using fibrobronchoscopy. In the current study we include patients with MPE and trapped lung after performing bronchoscopy to rule out endobronchial tumour obstruction.

There are two main causes for the formation of trapped lung in MPE. The leading cause is involvement of the visceral pleura by the multiple tumour metastases restricting the expansion of the lungs $(3,5,7)$. Lung carcinoma is the most frequent primary tumour location leading to metastatic disease in the 
pleural cavity. The second cause for trapped lung in MPE is fibrotic change of the visceral pleura. Higher fibrogenic potential is discussed in metastatic pleural effusions (8). In vivo and in vitro tests prove increased production of transforming growth factor by the tumour cells. The growth factor activates fibroblast proliferation and collagen matrix synthesis ( 9 , 10). These processes observed in pleural metastases lead to fibrotic reorganization of the visceral pleura. Trapped lung can result from radiation-induced fibrotic transformation of the visceral pleura $(3,7)$. There are cases of MPE with primary location - breast cancer and following postoperative radiation therapy.

Trapped lung is a cause for elevated values of the negative pressure in the pleural cavity. This leads to increased production and reduced absorption of pleural fluid $(11,3)$. Thus, the trapped lung itself acts as an additional indirect cause for the formation of a pleural effusion in metastatic disease of the pleura.

We diagnose trapped lung in MPE using plain chest radiography and CT of the thorax. The incomplete expansion of the lung after evacuating the pleural effusion is determined as pathognomonic radiologic sign for MPE with trapped lung - pneumothorax ex vacuo presented in this survey.

We need a method allowing direct assessment of the changes in the pleural cavity in order to reach definitive diagnosis of trapped lung. In this regard the video-assisted thoracoscopy provides exceptional possibilities. Apart from direct assessment of the pleural pathology and possibility for pleural biopsy, video-assisted thoracoscopy allows evaluation of the reexpansion of the lung. When trapped lung is established intraoperatively the reason for this condition should be determined as well diffuse metastatic involvement of the pleura or fibrotic changes of the visceral pleura.

Malignant pleural effusions are a sign of advanced stage of the disease with poor prognosis for the patients and life expectancy of 6 to 9 months $(1,12,13)$. With regard to the treatment plan, the main goal is achieving effective palliation of the clinical manifestations - reduction of dyspnea, pain relief, discomfort, better quality of life. The widely used method for chemical pleurodesis in the treatment of MPE with trapped lung, is inefficient as well as contraindicated $(3,7)$. The inability of the lung to expand fully leads to lack of contact between the two pleural membranes - an absolute condition for successful pleurodesis. In addition the presence of sclerosing agent in the pleural cavity will suppress even more the function of the lung as a result of the fibrotic transformation of the visceral pleura caused by the sclerosing agent.

Treatment options for MPE with trapped lung are: repeated pleural aspirations, permanent indwelling drainage catheter, pleural peritoneal shunting, pleurectomy with decortication (1, 2). Every single one of these methods has advantages and disadvantages as well as risks. It should be noted that not every method can be applied to all patients with MPE. In our survey the main method for treatment of MPE with trapped lung is insertion of permanent drainage tube. We use large-bore chest tubes sizes 26 to $32 \mathrm{Fr}$ in order to avoid blockage of the tube with coagula or fibrin materials. In these cases keeping the chest tube patent is more important than the discomfort of the patients caused by the large-bore tube (14). Evidence for the efficiency of permanent chest tube drainage is the improvement of dyspnea, visible on the radiography - lack of pleural effusion, pneumothorax ex vacuo, presence of very slight re-expansion of the trapped lung.

The most common complications of permanent chest tube drainage are inflammation of the soft tissues like acute cellutilis and infection of the pleural cavity - pleural empyema. The frequency of these two complications is comparable to other surveys on the same topic $(15,16,17)$.

\section{CONCLUSIONS}

1.Mandatory condition in the diagnostic process of trapped lung is exclusion of endobronchial tumour obstruction.

2.'Trapped lung' is definitively diagnosed through direct inspection of the affected pleural cavity performed with video-assisted thoracoscopy.

3.Insertion of permanent chest tube drainage in patients with MPE and trapped lung leads to effective palliation of the symptoms with short hospital stay and low frequency of possible complications.

\section{REFERENCES}

1. Antony, V. B et al. Management of malignant pleural effusions. - Eur. Respir. J., 18, 2001, 402-419.

2. L ig h t , R. W. Diagnosis and management of trapped lung. - UpToDate, 2004.

3. L ig h t , R. W. Pleural Diseases, $5^{\text {th }}$ ed. Lippincot, Baltimore, Williams and Wilkins, 2007.

4. Neragi, S. M. Malignant pleural effusion, current and evolving approaches for its 
diagnosis and management. - Lung Cancer, 54, 2006, 1-9.

5. Patlakas, G. et D. Bouros. Trapped lung. Int. Pleural Newsletter, 2005, № 1, 4-5.

6. Tan, C. et al. The evidence on the effectiveness of management for malignant pleural effusion: a systematic review. - Eur. J. Cardiothorac. Surg., 29, 2006, 829-838.

7. Shields, T. W. General Thoracic Surgery $6^{\text {th }}$ ed Lippincot, Williams and Wilkins. 2005, Chapter 68.

8. Doelken, P. et S. A. Sahn. Trapped lung. Respir. Crit. Care Med., 22, 2001, 631-634.

9. Border, A. W. et N. A. Noble. Transforming growth factor in tissue fibrosis. - N. Engl. J. Med., 331, 1994, 1286-1292.

10. L e e, Y. C. G. et B. K. Lane. Cytokines in pleural diseases. - In: Textbook of Pleural Diseases. R. W. Light et Y. C. G. Lee. (Eds.). London, Arnold Press, 2003' 63-89.

11. Doelken, P. et al. Pleural manometry Technique and clinical lmplications. Chest, 126, 2004, 1764-1769.
12. Arapis, K. et al. Thoracoscopic palliative treatment of malignant pleural effusions. Surg. Endosc., 20, 2006. 919-923.

13. Laisaar, T., V. Palmitse et T. Vooder. Life expectancy of patients with malignant pleural effusion treated with videoassited thoracoscopic talc pleurodesis. - Int. Cardiovasc. Thorac. Surg., 5, 2006, 307310.

14. Новаков, И. Блокиран бял дроб при малигнени плеврални изливи. Мед. Преглед 2009; 1: 57 - 62.

15. Park, D. et al. Use of indwelling pleural catheter compared with thorascopic talc pleurodesis in the management of malignant pleural effusions. - Am. Surg., 63, 2003, 198-202

16. Pien, G. W. et al. Use of implantable pleural catheter for trapped lung syndrome in patients with malignant pleural effusion. - Chest, 119, 2001, 1641 -1646.

17. Putnam, J. B. et al. Outpatient management of malignant pleural effusion by a chronic indwelling pleural catheter. - Ann. Thorac. Surg., 69, 2000, 369-375. 